\author{
Marie Sarazin \\ Agnès Michon \\ Bernard Pillon \\ Yves Samson \\ Alessandra Canuto \\ Gabriel Gold \\ Constantin Bouras \\ Bruno Dubois \\ Panteleimon Giannakopoulos
}

\section{Metabolic correlates of behavioral and affective disturbances in frontal lobe pathologies}

Received: 21 October 2002

Received in revised form: 11 February 2003

Accepted: 17 February 2003
Dr. A. Michon - Dr. A. Canuto - Dr. C. Bouras - Prof. Panteleimon Giannakopoulos (区)

Clinic of Geriatric Psychiatry

Department of Psychiatry

University of Geneva School of Medicine

1225 Chêne-Bourg

Geneva, Switzerland

Tel.: + 41-22/305-5001

Fax: + 41-22/305-5044

E-Mail:

Panteleimon.Giannakopoulos@medecine .unige.ch

Dr. G. Gold

Department of Geriatrics

University of Geneva School of Medicine

Geneva, Switzerland

Prof. P. Giannakopoulos

Service of Psychogeriatrics

University of Lausanne School

of Medicine

Lausanne, Switzerland

Dr. M. Sarazin · Dr. B. Pillon • Dr. B. Dubois Inserm E 007 and Federation

of Neurology

Salpêtrière Hospital

Paris, France
Dr. Y. Samson

Hospital Frédéric Joliot

CEA, Orsay, France

Supported by grant 31-52997.97 from the Swiss National Science Foundation, Bern, Switzerland (PG).

Abstract Objective Although previous studies have shown that the human frontal cortex is involved in the experience of emotions as well as in social behavior, data regarding the exact anatomical substrates of behavioral and affective deficits in frontal lobe pathologies are still scarce. The aim of this study was to investigate the metabolic correlates of these deficits in a group of non-selected consecutive patients with frontal lobe lesions. Patients and Methods Clinicometabolic correlations between several emotional and social parameters and metabolic patterns in the frontal cortex and amygdala were investigated in 32 patients with frontal lobe pathologies. The behavioral disturbances were evaluated using the Lhermitte's informant questionnaire. Regional cerebral glucose metabolism was measured with $\left[{ }^{18} \mathrm{~F}\right]$ fluorodeoxyglucose and high-resolution positron emission tomography.
Statistical analysis was performed using both single variable correlation and multiple regression analyses. Results Both single variable and multivariate analyses demonstrate that decreased regional glucose metabolism in the right medial area 10 was associated with apathy. There were also significant negative relationships between metabolism in the right orbitofrontal cortex and stereotypy and indifference to rules. Impulsiveness, personality disturbances and loss of emotional control were associated with decreased metabolism in the left amygdala. Conclusions In terms of clinicometabolic correlations, the present data support the implication of different functional anatomic systems in frontal lobe-related behavioral and affective disturbances. In particular, they imply that the classically described symptoms of impaired behavioral control may be related to right orbitofrontal cortex hypometabolism whereas impaired regulation of emotions may result from a functional damage of the left amygdala.

Key words amygdala brain . emotion $\cdot$ functional systems 


\section{Introduction}

Experimental work in both humans with brain lesions as well as in non-human primates support the notion of a functional specialization within the frontal cortex [10]. In a positron emission tomography (PET) study of the regional distribution of cerebral glucose metabolism in patients with frontal lobe damage, we previously showed a dissociation between the performance of executive tasks, related to the regional metabolism of the dorsolateral prefrontal cortex, and behavioral disturbances, associated with the regional metabolism of the orbitofrontal cortex in patients with frontal lobe lesions [39]. These findings indicate that impaired executive functions and social skill deficits are related to distinct metabolic patterns in patients with frontal lobe pathology.

However, behavioral changes associated with frontal lobe damage are highly heterogeneous. They include emotional and personality changes such as euphoria and irresponsibility, flat affect, decreased concern for the present or the future, impairment in the recognition of emotional expression, diminished empathy and social awareness, poor tolerance to frustration, increasing levels of aggressiveness and impulsiveness [7]. The human frontal cortex has been shown to be involved in the experience and appraisal of emotion and complex behavior as well as in primary and secondary mood disorders and social skills $[11,33]$. However, the precise neuroanatomical correlates of frontal lobe-related behavioral and affective disorders have yet to be defined.

The present study applied a clinico-metabolic approach, using PET, to identify anatomical areas related to behavioral and affective disturbances in a group of non-selected consecutive patients with frontal lesions. The underlying principle was that if a frontal lobe area were involved in a given behavioral or emotional process, a correlation should be observed between the score of patients in scales assessing this process and the level of metabolic activity in this area. This type of experimental design has been successfully used in earlier studies on patients with Alzheimer's disease or frontal lobe damage $[8,39]$.

\section{Patients and methods}

\section{Sample description}

The sample included 32 right-handed non-selected consecutive patients ( 20 women, $62.5 \pm 1.8$ years old; 12 men, $64.1 \pm 2.2$ years old) with frontal lobe lesions. Inclusion criteria were age between 25 and 75 years, documented frontal lobe pathology, informed written consent of the patient or appropriate surrogate, and the presence of an informant to assess behavioral abnormalities and changes in activities of daily living. All of the cases in the present report displayed severe deficits in executive functions that were assessed with an extensive neuropsychological battery which included Wisconsin and California card-sorting tests for conceptual elaboration [30], Stroop test for selective activation [16], lexical fluency [6], Trail Making Test [36], graphic series for cognitive flexibility [23], go-no go and conflicting instructions for motor control and inhibition [23], assessment of prehension, imitation and use behaviors for environmental dependency [21], and Grober and Buschke test for verbal learning and recall [17].

Exclusion criteria were the presence of severe psychiatric disorders such as bipolar illness, schizophrenia, and major depression according to DSM-IV diagnostic criteria, coexistence of non frontal brain lesions on magnetic resonance imaging (MRI), and contraindication for MRI or for arterial radial catheter. Among the patients studied, twelve presented with a focal frontal lobe lesion on MRI. Poststroke vascular lesions were present in nine cases: seven in the territory of anterior cerebral arteries (four in the right hemisphere, three in the left hemisphere) and two in the territory of the prefrontal branch of the left middle cerebral artery. Traumatic lesions confined to the frontal cortex were found in two cases (extensive bifrontal damage involving both the orbitofrontal and frontopolar regions) whereas in one case there was a post-surgical lesion (resection of a meningioma with subsequent damage of the orbitofrontal region [39]). In all these patients, lesions had occurred more than three months before the study. The remaining patients showed early frontal symptomatology, dysexecutive syndrome, decreased verbal fluency, and mild memory impairment and were classified as having frontotemporal dementia according to the Manchester and Lund diagnostic criteria [24].

\section{Behavioral assessment}

The behavioral disturbances were evaluated with the scale of Lhermitte and collaborators [21], administered to the patients' informants by the same experienced psychiatrist (P.G.) who was not aware of the PET results. The scale includes 80 randomly distributed questions designed to assess the differences between the premorbid and current state of the patient regarding the following 16 behavioral items: apathy, restlessness, stereotypy, impulsiveness, indifference, euphoria, disinterestedness, cheerfulness, dependence on social environment, indifference to rules, attention deficits, dependence on stimuli from the physical environment, programming deficits, personality disturbances, loss of intellectual and emotional control. Each item was assessed by five questions giving a score from 0 (absence of abnormality) to 5 (severe abnormality) for each item. The validity of this semi-structured interview for frontal lobe-related behavioral deficits has been previously established $[21,34,39]$. In particular, behavioral scores were significantly higher in patients with frontal lobe lesions than retrorolandic lesions [21] and in patients with subcorticofrontal dementia (progressive supranuclear palsy or Parkinson's disease) than in Alzheimer's disease [34]. Using this scale, our previous study in patients with frontal lobe lesions showed a strong correlation between most item scores and PET values in the orbitofrontal and frontopolar cortex bilaterally, whereas no significant correlation was found between behavioral data and metabolism at rest in other neocortical areas [39].

\section{Imaging procedures}

Brain imaging included a structural evaluation by MRI and a measurement of the glucose metabolism with $\left[{ }^{18} \mathrm{~F}\right]$ fluorodeoxyglucose $\left(\left[{ }^{18} \mathrm{~F}\right] \mathrm{FDG}\right)$ and high-resolution PET. MRI was performed with a 1.5$\mathrm{T}$ unit as previously described [39]. Briefly, patients were positioned in a plane parallel to the anterior-posterior commissures. Contiguous axial T1-weighted slices were taken with a slice interval of $3.37 \mathrm{~mm}$ to obtain MRI scans superimposable on the PET images. Coronal T2 images were also obtained in all cases. PET scans were performed on a high-resolution head-dedicated PET camera with a $5.8-\mathrm{mm}$ in plane and $5-\mathrm{mm}$ axial resolution, which provided 31 slices at $3.37-\mathrm{mm}$ in- 
tervals [5]. Just before the PET scan was performed, a 20-gauge 32$\mathrm{mm}$ Teflon cannula was placed in a radial artery under local anaesthesia to obtain blood measurements of $\left[{ }^{18} \mathrm{~F}\right]$ FDG and glucose concentrations. The patient's head was positioned in the bicommisural plane by aligning the skin marks drawn during the MRI with the crossed laser beam of the PET camera. Fifteen minutes were allowed to pass for patients to regain a comfortable state. All studies were conducted in a quiet, dimly lit environment with no background noise to minimize visual and auditory stimuli during the brain uptake phase. Patients were studied in an awake resting state with eyes closed. After an intravenous injection of $5 \mathrm{mCi}$ of $\left[{ }^{18} \mathrm{~F}\right] \mathrm{FDG}$, twenty four blood samples (one per 10 seconds during the first 2 minutes following injection) were collected from the radial artery to obtain blood measurements of $\left[{ }^{18} \mathrm{~F}\right] \mathrm{FDG}$ and glucose concentration. Image acquisition started 30 minutes after this injection and ended 20 minutes later [39]. Local cerebral metabolic rate for glucose (rCMRGlu expressed in $\mathrm{mg} / \mathrm{min} / 100 \mathrm{~g}$ tissue) was then calculated according to the model of Reivich and collaborators for human PET studies [37]. This study was approved by the Pitié-Salpêtrière Hospital Ethical Committee.

PET and MRI scans were transferred to a workstation and superimposed using automatic three-dimensional registration software [25]. Regions of interest (ROIs) were drawn manually on the axial images of MRI with the help of a stereotactic atlas [40] (Fig. 1). The segmentation of the cortical areas was based on Brodmann's nomencla- ture. Twenty ROIs were defined in the prefrontal cortex (bilaterally areas 8,9 , and 10 further divided into medial and lateral parts; areas 45 as a ventrolateral region; area 47 ; areas $11,12,13$ and 14 pooled as a single orbitofrontal region; areas 24 and 32 pooled as a single anterior cingulate area), and eight ROIs outside the frontal lobes were also considered (bilaterally area 7 , visual area V1, hippocampus, amygdala). ROIs drawn on the MRI scans were then transposed onto the PET scans and regional CMRGlu were calculated for each ROI.

\section{Statistical analysis}

Relationships between PET values in ROIs and behavioral item scores were first assessed by single variable correlation analysis using Spearman's coefficient $\left(r_{s}\right)$. For this analysis, correction for multiple comparisons was made by adopting a significance threshold value of $\mathrm{p}<0.005$. In addition, maximum likelihood ordered logistic regression analyses were built to examine whether metabolic data can predict the presence of a given behavioral disturbance. PET values in ROIs which significantly correlated with behavioral scores in the single variable analysis as well as possible confounding factors such as age and Mini Mental State Examination scores [13] were used as independent variables in this model with each behavioral score as the dependent variable. This method can evaluate the relationship be-
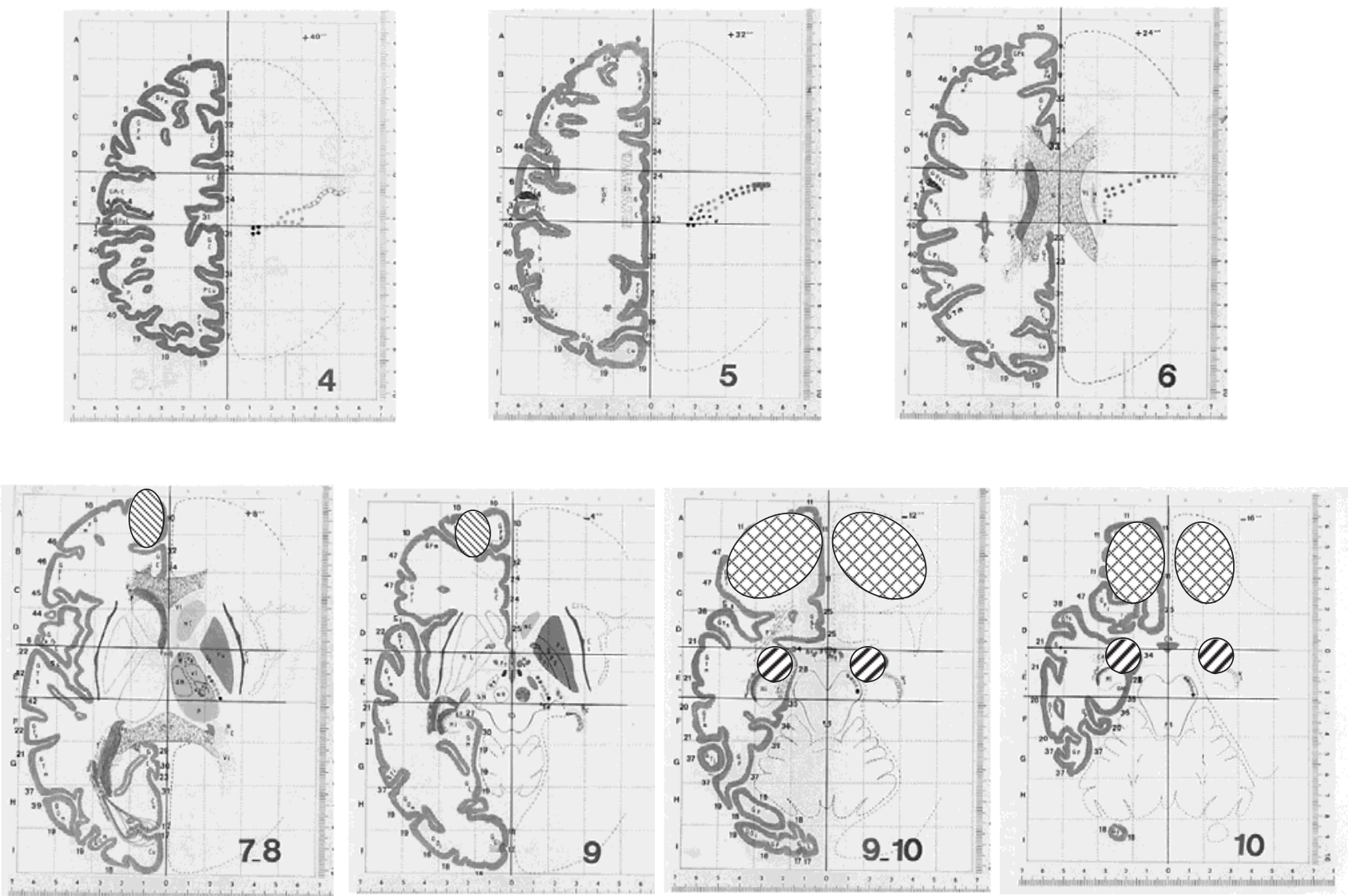

Fig. 1 Schematic representation of ROI on a Talairach brain. Dashed areas represent areas of interest where behavioral dysfunctions are correlated with local metabolic impairment.

apathy/right medial area 10

Impaired behavioral control/left and right orbitofrontal cortex

Impaired emotional control/left and right amygdala 
tween an ordinal outcome variable and several independent variables [14]. All statistical analyses were performed using Stata ${ }^{\mathrm{tm}}$ software Release 5 (College Station, TX).

\section{Results}

The distribution of metabolic values in frontal lobe and control areas in the present series is illustrated in Fig. 2. Data from single variable analyses are summarized in Tables 1 and 2. A similar pattern of correlations was found in both hemispheres, although the number of significant correlations was higher in the right one. In both hemispheres, statistically significant correlations were all negative. Significant correlations were found between behavioral deficits and glucose metabolism in the orbitofrontal cortex and area 10 bilaterally: they concern apathy (with a correlation coefficient that reaches 0.93 in the right medial area 10), stereotypy, euphoria, disinterestedness, dependence on the social and physical environment, indifference to rules, attention and programming deficits and loss of intellectual control. Metabolic values in amygdala correlated highly with impulsiveness, personality disturbances and loss of emotional control. No significant correlations were found between these behavioral items and regional metabolism values in frontal areas. Metabolic values in areas 8 , 9 , and 47, ventrolateral frontal and anterior cingulate cortex as well as in control areas $7 \mathrm{~V} 1$ and hippocampus
Table 1 Statistically significant correlations betwen behavioral items and PET values in the left hemisphere

\begin{tabular}{lll}
\hline Area & Spearman's coefficient & Statistical significance \\
\hline $\begin{array}{ll}\text { Apathy } \\
\text { 10M }\end{array}$ & -0.74 & $\mathrm{p}<0.005$ \\
OFL & -0.80 & $\mathrm{p}<0.001$ \\
$\begin{array}{l}\text { Impulsiveness } \\
\text { Amygdala }\end{array}$ & -0.75 & $\mathrm{p}<0.005$ \\
$\begin{array}{l}\text { Indifference to rules } \\
\text { OFL }\end{array}$ & -0.71 & $\mathrm{p}<0.005$ \\
$\begin{array}{l}\text { Dependence on the } \\
\text { physical environment } \\
\quad \text { 10L }\end{array}$ & -0.69 & $\mathrm{p}<0.005$ \\
$\begin{array}{l}\text { Personality disturbances } \\
\text { Amygdala }\end{array}$ & -0.78 & $\mathrm{p}<0.001$ \\
$\begin{array}{l}\text { Loss of emotional control } \\
\text { Amygdala }\end{array}$ & -0.79 & $\mathrm{p}<0.001$ \\
\hline
\end{tabular}

Results represent Spearman's correlation coefficients between behavioral item scores and regional glucose metabolism in each area. $10 \mathrm{~L}$ lateral part of area 10; $10 M$ medial part of area 10; OFL orbitofrontal cortex. See text for details

did not display any significant correlation with behavioral items in either hemisphere.

In multivariable models, decreased regional glucose metabolism in right medial area 10 was a strong predictor of apathy explaining $57 \%$ of the variability in apathy scores [regression coefficient: $-2.32,95 \%$ Confidence intervals: $(-4.60,-1.90), \mathrm{p}<0.005$, Fig. 1$]$.

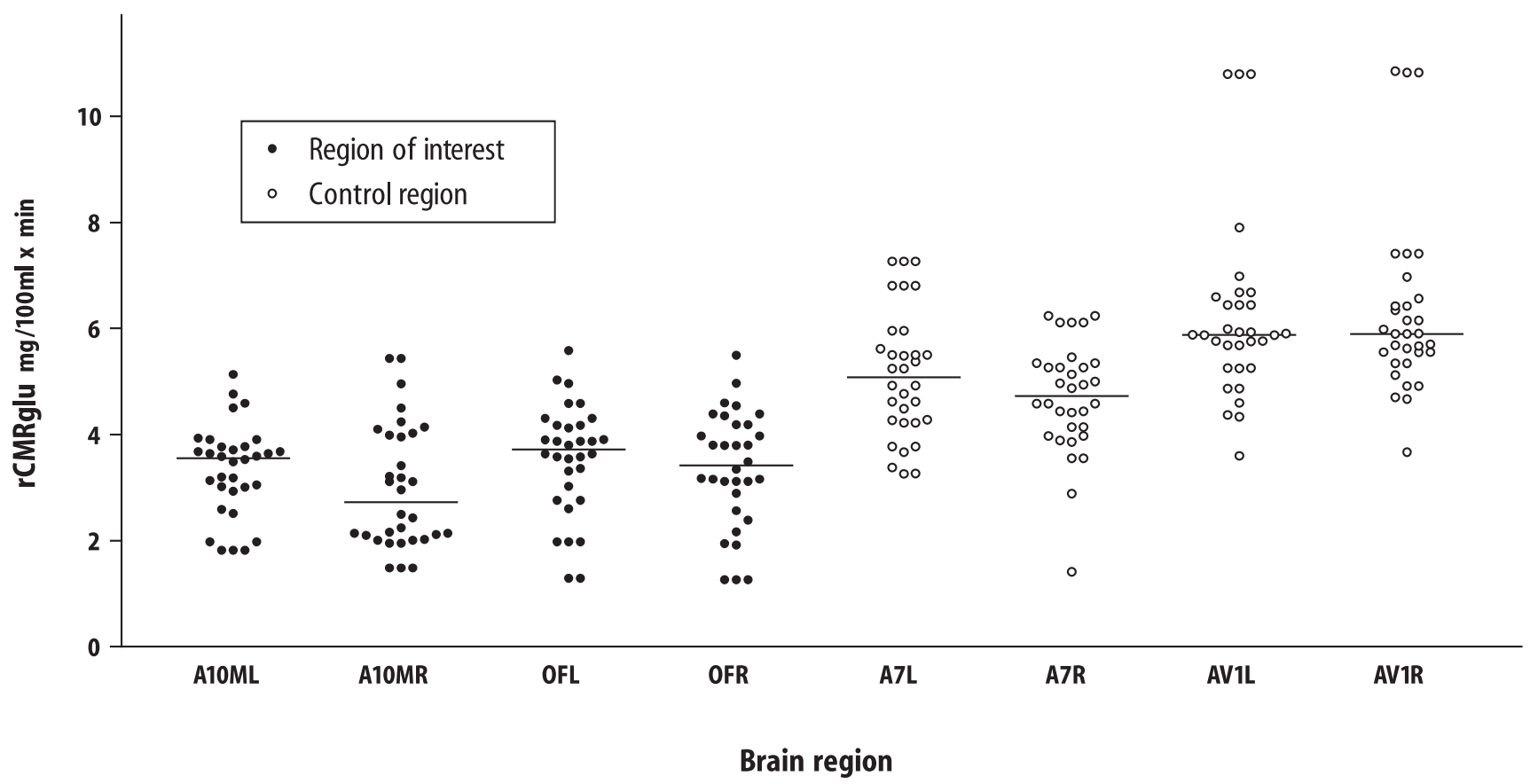

Fig. 2 Distribution of regional glucose metabolic values in frontal lobe subdivisions and control areas. Note the higher values in areas 7 and $\mathrm{V} 1$ compared to those observed in areas involved in behavioral deficits. A10ML left medial area 10; A10MR right medial area 10; OFL left orbitofrontal cortex; OFR right orbitofrontal cortex; $A 7 L$ left area 7; $A 7 R$ right area 7; AV1L left area V1; AV1R right area V1 
Table 2 Statistically significant correlations betwen behavioral items and PET values in the right hemisphere

\begin{tabular}{|c|c|c|}
\hline Area & Spearman's coefficient & Statistical significance \\
\hline \multicolumn{3}{|l|}{ Apathy } \\
\hline $10 \mathrm{M}$ & -0.93 & $p<0.001$ \\
\hline $10 \mathrm{~L}$ & -0.69 & $p<0.005$ \\
\hline \multicolumn{3}{|l|}{ Stereotypy } \\
\hline OFL & -0.68 & $p<0.005$ \\
\hline \multicolumn{3}{|l|}{ Euphoria } \\
\hline $10 \mathrm{M}$ & -0.70 & $p<0.005$ \\
\hline $10 \mathrm{~L}$ & -0.74 & $p<0.005$ \\
\hline OFL & -0.74 & $p<0.005$ \\
\hline \multicolumn{3}{|c|}{ Disinterestedness } \\
\hline $10 \mathrm{M}$ & -0.75 & $p<0.005$ \\
\hline $10 \mathrm{~L}$ & -0.69 & $\mathrm{p}<0.005$ \\
\hline OFL & -0.76 & $p<0.001$ \\
\hline \multicolumn{3}{|c|}{$\begin{array}{l}\text { Dependence on the } \\
\text { social environment }\end{array}$} \\
\hline $10 \mathrm{M}$ & -0.71 & $p<0.005$ \\
\hline OFL & -0.77 & $p<0.001$ \\
\hline \multicolumn{3}{|c|}{ Indifference to rules } \\
\hline OFL & -0.74 & $p<0.005$ \\
\hline \multicolumn{3}{|c|}{ Attention deficits } \\
\hline OFL & -0.79 & $p<0.001$ \\
\hline \multicolumn{3}{|c|}{$\begin{array}{l}\text { Dependence on the } \\
\text { physical environment }\end{array}$} \\
\hline $10 \mathrm{M}$ & -0.80 & $p<0.001$ \\
\hline $10 \mathrm{~L}$ & -0.77 & $p<0.001$ \\
\hline OFL & -0.86 & $p<0.001$ \\
\hline \multicolumn{3}{|c|}{ Programming deficits } \\
\hline $10 \mathrm{M}$ & -0.70 & $p<0.005$ \\
\hline OFL & -0.78 & $p<0.001$ \\
\hline \multicolumn{3}{|c|}{ Personality disturbances } \\
\hline Amygdala & -0.74 & $p<0.005$ \\
\hline \multicolumn{3}{|c|}{ Loss of intellectual control } \\
\hline $10 \mathrm{M}$ & -0.82 & $p<0.001$ \\
\hline 10L & -0.73 & $p<0.005$ \\
\hline OFL & -0.85 & $p<0.001$ \\
\hline
\end{tabular}

Results represent Spearman's correlation coefficients between behavioral item scores and regional glucose metabolism in each area. $10 \mathrm{~L}$ lateral part of area 10; $10 M$ medial part of area 10; OFL orbitofrontal cortex. See text for details

Significant relationships were found between decreased glucose metabolism in the right orbitofrontal cortex and stereotypy. This metabolic variable accounted for $43 \%$ of the variability in stereotypy scores [regression coefficient: $-1.63,95 \%$ Confidence intervals: $(-2.08,-0.98), \mathrm{p}<0.01]$. Decreased glucose metabolism in the right $\left[R^{2}=0.63\right.$, regression coefficient: $-1.82,95 \%$ Confidence intervals: $(-2.37,-1.27), \mathrm{p}<0.01]$ and left orbitofrontal cortex $\left[\mathrm{R}^{2}=0.73\right.$, regression coefficient: $-1.92,95 \%$ Confidence intervals: $(-2.34,-1.60), \mathrm{p}<0.01$ ] was also associated with indifference to rules (Fig. 1). Because inappropriate behavioral control was present in these two deficits we grouped them under the term of "impaired behavioral control".

Finally, hypometabolism in left amygdala could predict scores of impulsiveness $\left[R^{2}=0.38\right.$, regression coef- ficient: $-2.34,95 \%$ Confidence intervals: $(-3.95,-1.66)$, $\mathrm{p}<0.01]$, personality disturbances $\left[\mathrm{R}^{2}=0.28\right.$, regression coefficient: $-2.36,95 \%$ Confidence intervals: $(-3.31$, $-1.40), \mathrm{p}<0.01]$ and loss of emotional control $\left[\mathrm{R}^{2}=0.47\right.$, regression coefficient: $-2.68,95 \%$ Confidence intervals: $(-3.44,-1.91), \mathrm{p}<0.005]$. A weak but significant association was also found between decreased metabolism in right amygdala and personality disturbances $\left[\mathrm{R}^{2}=0.23\right.$, regression coefficient: $-1.90,95 \%$ Confidence intervals: $(-2.26,-1.22), \mathrm{p}<0.05$, Fig. 1]. Because these items concerned all emotional and affective changes, we grouped them under the term of "impaired emotional control".

No statistically significant relationships were found between the remaining behavioral items and PET values with either multivariable analysis. Importantly, there was no significant correlation between patterns of hypometabolism and structural changes (documented by MRI) in patients with high scores for apathy, "impaired behavioral control" and "impaired emotional control" supporting the added value of PET data in predicting behavioral deficits. In agreement with our previous work [39], there were no statistically significant correlations between neuropsychological test performances and behavioral ratings in the present study. Confirming our previous observations [39], neuropsychological data in the present series showed a strong correlation with PET values in dorsolateral frontal areas 8 and 9 but not in medial area 10, orbitofrontal cortex and amygdala (data not shown).

\section{Discussion}

Strengths of the present study are the large series of prospectively studied cases with various frontal lobe pathologies, the fact that behavioral data were collected by a single experienced psychiatrist, blinded to other findings, as well as the rigorous statistical methodology including both single variable correlation analysis corrected for multiple comparisons and multivariable models. The single variable analysis revealed a much higher number of statistically significant correlations in the right than in the left frontal cortex. This finding parallels several earlier studies suggesting a hemispheric asymmetry in the processing of emotion and social behavior in the frontal cortex. For instance, the right hemisphere is more active than the left during the processing of facial emotion [29], and patients with right frontal cortex lesions show difficulties in addressing emotional communication information [38]. Based on these observations, it has been proposed that the functional anatomic organization of emotional communication in the right hemisphere mirrors that of propositional language in the left hemisphere [38]. Importantly, a recent functional imaging study in patients with frontotemporal dementia showed that the right frontal lobe also plays 
a key role in maintaining the essential qualities distinguishing one person from another referred to as self concept [26].

Apathy scores in this series were associated with decreased metabolism in right medial area 10 [31]. This observation parallels several recent lines of evidence indicating that the ventromedial system participates in the intentional prospective control of behavior related to internal information [15]. In particular, the medial frontal region may be primarily involved in self-initiated actions from an inner source as proposed by Laplane and Dubois in their description of the syndrome of auto-activation deficit [19]. Importantly, in a recent fMRI study, Pochon and collaborators also reported an activation of medial area 10 after reward during working memory activation in healthy controls and proposed that this area plays a specific role in monitoring the reward value of ongoing cognitive processes [35]. The specific association described here between decreased metabolism in the right medial area 10 and apathy agrees with earlier observations of Craig and collaborators who showed a prefrontal hypoperfusion in apathetic patients with Alzheimer's disease and postulated that apathy results from a damage of the medial frontal-subcortical circuit in this condition $[8,9]$.

The present results also provide new insights into the metabolic basis of complex behavioral and emotional phenomena classically attributed to the frontal lobe. In respect to behavioral disturbances, they support an association between hypometabolism in the orbitofrontal cortex and "impaired behavioral control" in frontal lobe pathologies $[9,31,41]$. They also indicate a strong relationship between left amygdala hypometabolism and "impaired emotional control" symptoms such as impulsiveness, personality changes (including irritability, increased anger, and frequent loss of patience) and loss of emotional control. These observations should be interpreted in the light of current hypotheses regarding the role of these areas in social behavior. The right orbitofrontal cortex is thought to regulate stimulus-reinforcement associated learning, and it has been recently demonstrated that macaques with lesions in this area display aberrant behaviors when reinforcement contingencies change [38]. Moreover, despite intact general cognitive and executive functions, patients with damage of the right orbitofrontal cortex exhibit severe antisocial behavior characterized by reduced empathy and marked difficulties in a moral/conventional distinction task $[4,7,22]$. Both animal and functional imaging studies have shown that the left amygdala is mainly involved in the extraction of affective content from visual stimuli $[2,3,18,20,27,28]$. In humans, lesions of this area can lead to selective deficits in the recognition of fearful facial expressions and impaired fear conditioning $[1,28$, 33]. Furthermore, early left amygdala damage is associated with severe impairment in the ability to predict and understand other people's mental states [12]. As postulated by Adolphs and collaborators, this area may be required for accurate social judgments of other individuals on the basis of their facial appearance [1]. One attractive scenario is that a dysfunction of these areas might participate in the inadequate social interactions that characterize frontal lobe pathologies by inducing an inability to perceive other's emotional states and modify behavior in response to the social feedback.

In conclusion, our results indicate that separable and probably complementary anatomofunctional systems may intervene in control of behavior and emotion in frontal lobe pathologies. Detailed correlative analyses in large prospective series including functional and structural imaging, neuropathological assessment as well as evaluation of complex cognitive functions such as processing of mental states would be useful for defining better the neuroanatomical substrates of social behavior deficits in these conditions.

\section{References}

1. Adolphs R, Tranel D, Damasio H, Damasio AR (1995) Fear and the human amygdala. J Neurosci 15: 5879-5891

2. Adolphs R, Tranel D, Damasio AR (1988) The human amygdala in social judgment. Nature 393:470-474

3. Aggleton JP (1993) The contribution of the amygdala to normal and abnormal emotional states. Trends Neurosci 16: 328-333

4. Bechara A, Damasio H, Damasio AR (2000) Emotion, decision making and the orbitofrontal cortex. Cereb Cortex 10:295-307

5. Bendriem B (1991) Quantitative evaluation of a new brain tomograph: the ECAT 953B/31. J Nucl Med 32:1063
6. Benton AL (1968) Differential behavioural effects in frontal lobe disease. Neuropsychologia 6:53-60

7. Blair RJR, Cipolotti L (2000) Impaired social response reversal. A case of "acquired sociopathy". Brain 123: 1122-1141

8. Craig AH, Cummings JL, Fairbanks L, Itti L, Miller BL, Li J, Mena I (1996) Cerebral blood flow correlates of apathy in Alzheimer's disease. Arch Neurol 53:1116-1120

9. Cummings JL (1995) Anatomic and behavioral aspects of frontal-subcortical circuits. Ann N Y Acad Sci 769:1-13

10. Cummings JL (1993) Frontal-subcortical circuits and human behavior. Arch Neurol 50:873-880
11. Damasio AR, Tranel D, Damasio H (1991) Somatic parkers and the guidance of behavior: theory and preliminary testing. In: Llevin HS, Eisenberg HM, Benton AL (eds) Frontal lobe function and dysfunction, Oxford University Press, Oxford, pp 271-279

12. Fine C, Lumsden J, Blair RJ (2001) Dissociation between 'theory of mind' and executive functions in a patient with early left amygdala damage. Brain 124: 287-298

13. Folstein MF, Folstein SE, McHugh PR (1975) Mini-mental state: a practical method for grading the cognitive state of patients for the clinician. J Psychiatry Res 12:189-198 
14. Gold G, Kovari E, Corte G, Herrmann FR, Canuto A, Bussiere T, Hof PR, Bouras C, Giannakopoulos P (2001) Clinical validity of $A \beta$-protein deposition staging in brain aging and Alzheimer disease. J Neuropathol Exp Neurol 60:946-952

15. Goldberg G (1987) From intent to action: evolution and function of the premotor system of the frontal lobe. In: Perecman E (ed) The frontal lobes revisited. The IRBN Press, New York, pp 273-306

16. Golden CJ (1978) Stroop color and word test, Chicago: Stoelting Company

17. Grober E, Buschke H (1987) Genuine memory deficits in dementia. Dev Neuropsychol 3:13-36

18. Irwin W, Davidson RJ, Lowe MJ, Mock BJ, Sorenson JA, Turski PA (1996) Human amygdala activation detected with echo-planar functional magnetic resonance imaging. Neuroreport 7: 1765-1769

19. Laplane D, Dubois B (2001) Auto-activation deficit : a basal ganglia related syndrome. Mov Dis 16:810-814

20. Ledoux JE (2000) Emotion circuits in the brain. Annu Rev Neurosci 23: 155-184

21. Lhermitte F, Pillon B, Serdaru M (1986) Human autonomy and the frontal lobes. Part 1: Imitation and utilization behaviors: a neuropsychological study of 75 patients. Ann Neurol 19:41-60

22. Lough S, Gregory C, Hodges JR (2001) Dissociation and executive function in frontal variant frontotemporal dementia. Neurocase 7:123-130

23. Luria AR (1966) Higher cortical functions in man, Basic Books, New York

24. Manchester and Lund Groups (1994) Clinical and neuropsychological criteria for frontotemporal dementia. J Neurol Neurosurg Psychiatry 57: 416-418
25. Mangin JF, Frouin V, Bloch I, Bendriem B, Lopez-Krahe J (1994) Fast nonsupervised $3 \mathrm{D}$ registration of $\mathrm{PET}$ and MR images of the brain. J Cereb Blood Flow Metab 14:749-762

26. Miller BL, Seeley WW, Mychack P, Rosen HJ, Ismael M, Boone K (2001) Neuroanatomy of the self, evidence from patients with frontotemporal dementia. Neurology 57:817-821

27. Morris JS, Friston KJ, Buchel C, Frith CD, Young AW, Calder AJ, Dolan RJ (1998) A neuromodulatory role for the human amygdala in processing emotional facial expressions. Brain 121: 47-57

28. Morris JS, Frith CD, Perrett DI, Rowland D, Young AW, Calder AJ, Dolan RJ (1996) A differential neural response in the human amygdala to fearful and happy facial expressions. Nature 383: 812-815

29. Nakamura K, Nakamura K, Kawashima $\mathrm{R}$, Ito K, Sugiura M, Kato T, Nakamura A, Hatano K, Nagumo S, Kubota K, Fukuda H, Kojima S (1999) Activation of the right inferior frontal cortex during assessment of facial emotion. J Neurophysiol 82:1610-1614

30. Nelson HE (1976) A modified card sorting test sensitive to frontal lobe defects. Cortex 12:313-324

31. Niedermeyer E (1998) Frontal lobe functions and dysfunctions. Clin Electroencephalogr 29:79-90

32. Paradiso S, Robinson RG, Andreasen NC, Downhill JE, Davidson RJ, Kirchner PT, Watkins GL, Ponto LL, Hichwa RD. Emotional activation of limbic circuitry in elderly normal subjects in a PET study. Am J Psychiatry 154: 384-389

33. Phelps EA, O'Connor KJ, Gatenby JC, Gore JC, Grillon C, Davis M (2001) Activation of the left amygdala to a cognitive representation of fear. Nature Neurosci 4:437-441
34. Pillon B, Dubois B, Lhermitte F, Agid Y (1986) Heterogeneity of cognitive impairment in progressive supranuclear palsy, Parkinson's disease, and Alzheimer's disease. Neurology 36: 1179-1185

35. Pochon JB, Levy R, Fossati P, Lehericy S, Poline JB, Pillon B, Le Bihan D, Dubois B (2002) The neural system that bridges reward and cognition in humans: An fMRI study. Proc Natl Acad Sci U S A 99:5669-5674

36. Reitan RM (1958) Validity of the Trail Making test as an indication of organic brain damage. Percept Mot Skills 8: 271-276

37. Reivich M, Kuhl D, Wolf A, Greenberg J, Phelps M, Ido T, Casella V, Fowler J, Hoffman E, Alavi A, Som P, Sokoloff L (1979) The mapping of functional activity in the human brain with $18 \mathrm{~F}-\mathrm{r}$ fluorodeoxyglucose technique. Workshop in cerebrovascular disease. Stroke 10:484-491

38. Rolls ET (2000) The orbitofrontal cortex and reward. Cereb Cortex 10: 284-294

39. Sarazin M, Pillon B, Giannakopoulos P, Rancurel G, Samson Y, Dubois B (1998) Clinicometabolic dissociation of cognitive functions and social behavior in frontal lobe lesions. Neurology 51: 142-148

40. Talairach J, Tournoux P (1988) A coplanar stereotaxic atlas of a human brain. Stuttgart: Thieme

41. Tekin S, Mega MS, Masterman DM, Chow T, Garakian J, Vinters HV, Cummings JL (2001) Orbitofrontal and anterior cingulate cortex neurofibrillary tangle burden is associated with agitation in Alzheimer disease. Ann Neurol 49:355-361 